\title{
PERCEPCIÓN DE LOS ALUMNOS SOBRE LA UTILIZACIÓN DE REALIDAD AUMENTADA EN LA ASIGNATURA DE DISEÑO DE HORMIGÓN REFORZADO
}

Gláucia Nolasco de Almeida Mello-gnamello@ pucminas.br

Pontifícia Universidade Católica de Minas Gerais - Departamento de Engenharia Civil

Rua Dom José Gaspar, 500 Prédio 3 - sala 102, Coração Eucarístico

CEP 30535-901 - Belo Horizonte - MG

Resumen: Para el Ingeniero Civil, una de sus principales actividades es el desarrollo de diseño y, de esta manera, su implicación con dibujos y representación de estructuras es intensa. Así, la habilidad de visualización espacial y rotación mental es especialmente importante para ese profesional. De esa manera, la motivación general de esta investigación fue planear y desarrollar actividades utilizándose aplicación para móviles con los recursos de la realidad aumentada, para las disciplinas de dibujos de hormigón reforzado en el curso de Ingeniería Civil. Se desarrolló cuatro actividades utilizándose los recursos de realidad aumentada con la aplicación Sketchfab. Treinta y ocho alumnos hicieron las actividades y respondieron a un cuestionario que fue utilizado como instrumento para evaluar la percepción de los alumnos acerca de la utilidad, facilidad de uso, motivación e intención de uso de los recursos para el aprendizaje de los contenidos de la asignatura. Casi todos los alumnos creen que los recursos empleados son muy relevantes para el aprendizaje, hacen el aprendizaje más divertido y facilitan la visualización de los detalles de las estructuras. En ese contexto, se concluye que, mientras haga mucho que investigar aun, la $R A$ es un recurso importante para mejorar la habilidad de visualización espacial de los estudiantes de ingeniería y por consecuencia el desempeño de estructuras.

Descriptores: Diseño estructural. Hormigón reforzado. Visualización espacial. Realidad aumentada.

\section{INTRODUCCIÓN Y MARCO TEÓRICO}

Las edificaciones de estructuras de hormigón, acero, madera o de otro material, con sus arreglos complejos de los elementos estructurares, son comúnmente representados en un conjunto de dibujos en dos dimensiones. En los dibujos son indicadas la cantidad, la longitud y el diámetro de los aceros y, también, sus posiciones dentro de los elementos estructurales, o sea, toda la información necesaria para la ejecución exitosa de la estructura. En las universidades se han utilizado los dibujos y las imágenes estáticas, bidimensional (2D) o tridimensional (3D), para transmitir la complejidad de estos arreglos y sus modos de interacción. De acuerdo con Fogarty, Mccormick y El-Tawil (2018), la representación 2D requiere que los estudiantes construyan una imagen del elemento estructural o conjunto de varios elementos con información y experiencia limitadas. Además, combinado eso con la falta de énfasis en dibujos bidimensionales de las estructuras tridimensionales en los cursos ingeniería civil pueden obstaculizar la capacidad de los estudiantes de visualización espacial de los arreglos en dos dimensiones. Visualizar arreglos espaciales y complejos puede ser un desafío para algunas personas aún más cuando hay la deformación o el movimiento de estos arreglos en varios escenarios de carga u otros estímulos externos. De acuerdo con Maier (1994 apud SORBY, 2001), para profesiones tecnológicas como por ejemplo la ingeniería, la habilidad de visualización espacial (HVE) y rotación mental es especialmente importante. 


\subsection{Habilidad de Visualización Espacial (HVE)}

Lin (2016) ha presentado algunas pruebas para evaluar la HVE de los estudiantes. En ese conjunto de pruebas se encuentra la de la Universidad de Purdue - Purdue Spatial Visualization Test (PSVT), que fue utilizada por Sorby y Veurink (2012) para evaluar la capacidad de visualización espacial de estudiantes estadounidenses y de otras partes del mundo. En la investigación de estos autores, ellos concluyen que las diferencias culturales en la educación preuniversitaria entre los estudiantes estadounidenses y particularmente de aquellos países de los cuales son reclutados a la mayoría de los estudiantes internacionales en Estados Unidos, son probablemente un factor importante que caracterizan las habilidades espaciales poco desarrolladas. Segil et al. (2017) también investigaron la capacidad de visualización espacial de estudiantes de varias partes del mundo en la universidad estadounidense. Los autores (SEGIL et al., 2017) han propuesto un workshop para los estudiantes que no han obtenido la media necesaria en la prueba PSVT y, ellos concluyeron que el entrenamiento fue efectivo, pero no suficiente.

Con la finalidad de mejorar HVE en el alumno, Mello, Maia y Calixto (2016) han planeado y desarrollado un sitio en la internet para la enseñanza de proyectos de hormigón reforzado. Entre otras actividades el sitio tenía una aplicación web para calcular elementos estructurales (vigas y columnas) de hormigón reforzado. Por medio de la aplicación los alumnos podrían interactuar con el programa informático y determinar la rotación de la estructura. La aplicación fue desarrollada en lenguaje de programación Java, con recursos Java 3D. Fogarty, Mccormick y El-Tawil (2018) han investigado el uso de herramientas de realidad virtual para ayudar a los estudiantes a comprender lo complejo del concepto de pandeo en estructuras de ingeniería. Este estudio de métodos mixtos analiza los exámenes previos y posteriores que cubren temas que requieren habilidades de visualización espacial, así como también encuestas y entrevistas a los estudiantes que usan las herramientas de realidad virtual. Los resultados cuantitativos indican que los estudiantes pueden identificar y visualizar los modos de pandeo de forma más precisa después de la experiencia de realidad virtual. Cualitativamente, los estudiantes expresan una mejor comprensión, mayor entusiasmo por el tema y mayor deseo de que otros temas sean presentados usando herramientas de realidad virtual.

\subsection{Realidad Aumentada}

Segundo Azuma (1997), señala que la realidad aumentada es cualquier sistema que tenga las tres siguientes características: combina el mundo real y virtual, es interactivo en tiempo real y está registrado en tres dimensiones. Realidad aumentada (RA) es un campo de investigación en ciencias de la computación que combina realidad y datos digitales, esto es, emplea visión por computadora, procesamiento de imágenes y técnicas gráficas para fusionar contenido digital en el mundo real. Cabero y Barroso (2016) en la presentación de las posibilidades de utilización de RA en la educación mostraron algunas ventajas del uso de ese recurso: (1) ayudan en la adquisición del conocimiento que se vuelve esencial para relacionar y comprender los conceptos aprendidos por medio de la interacción con los recursos de RA con el entorno real; (2) promueven un aprendizaje más personalizado de modo que cada alumno pueda progresar al ritmo marcado por sus propias capacidades e intereses y; (3) propician a los estudiantes un mayor nivel de interacción y exploración tanto sobre información como sobre objetos.

\subsection{Modelo TAM para Evaluación de la Percepción del Alumno}

El modelo de aceptación de tecnología (Technology Acceptation Model - TAM) fue adaptado de la teoría de la acción razonada (Theory of Reasoned Action - TRA) por Davis en 
1986 (ABDULLAH y WARD, 2016) para explicar el comportamiento de una persona para la adopción de tecnología (DAVIS, 1989). En el modelo TAM, se proponen algunas variables para delinear el impacto de los factores externos en las dos percepciones principales del usuario en relación con el uso de la tecnología: (1) la facilidad de uso percibida y (2) la utilidad percibida. Según Davis (1989), el primero influye directamente en el segundo y ambos influyen en las actitudes positivas o negativas de los usuarios con respecto al uso de la tecnología. La actitud hacia el uso de la tecnología influye en la intención de comportamiento de usar la tecnología. Y, la intención de comportamiento de usar la tecnología determina el uso real (DAVIS, 1989; ABDULLAH y WARD, 2016). La Figura 1 muestra el esquema del modelo TAM, según Davis (1989).

El modelo TAM propone que sean identificadas las diferentes variables externas que pueden incidir en la utilidad y la facilidad de uso percibidas por los usuarios de tecnología. De acuerdo con Cabero y Pérez (2018), aún que diferentes estudios han sugerido nuevas propuestas y el modelo ha evolucionado a lo largo del tiempo, él permanece esencialmente compuesto de un conjunto simple de variables identificadas, como en la formulación original, que se presentan como robustas y confiables.

Figura 1 - Modelo TAM

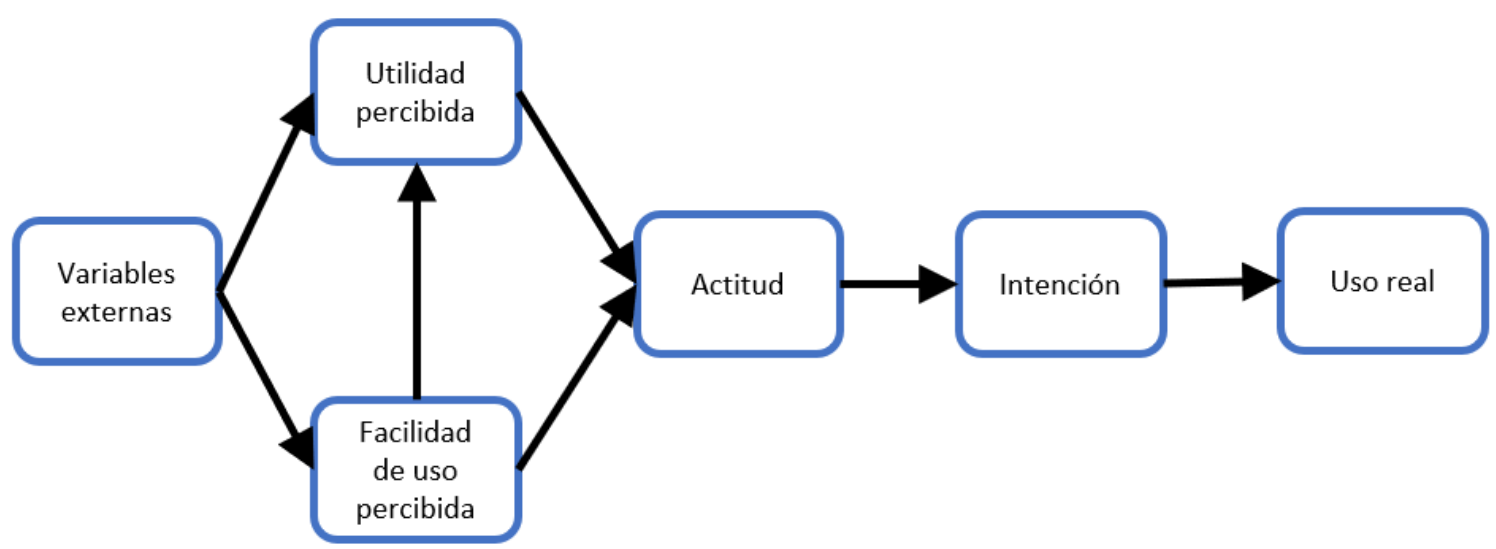

Fuente: Adaptado de Al-Emran, Mezhuyev y Kamaludin (2018)

\subsection{Objetivos}

Aunque los investigadores han evidenciado los factores principales que comprometen el proceso de enseñanza y aprendizaje in ingeniería (MOLYNEAUX et al. 2007; MELLO, 2016), en especial la dificultad que tienen los estudiantes de ingeniería en la visualización espacial (SORBY, 2001; MELLO, MAIA y CALIXTO, 2016; FOGARTY, MCCORMICK y EL-TAWIL; 2018), hay mucho que hacer para mejorar la HVE de los estudiantes en la enseñanza de ingeniería de estructuras. En este escenario, la motivación general de esta investigación es planear y desarrollar actividades utilizándose aplicación para móviles con los recursos de la realidad aumentada, para las disciplinas de hormigón reforzado en el curso de Ingeniería Civil.

\section{METODOLOGÍA}

El presente proyecto es una investigación descriptiva con enfoque cualitativo para validar la metodología y las herramientas elegidas para las actividades desarrolladas con el objetivo 
de establecer si el uso de la RA motiva y ayuda el aprendizaje del alumno en las asignaturas de hormigón reforzado.

Fue elegida la asignatura de Expresión Gráfica en el octavo año de Ingeniería Civil de la Pontificia Universidad Católica de Minas Gerais en Brasil. En esa asignatura los alumnos no solo deben interpretar los dibujos hechos como también deben representar, en dibujos 2D, toda la estructura de hormigón reforzado ya calculada. Fueran desarrollados cuatro modelos 3D para RA por medio de Sketchup (https://www.sketchup.com) y, las cuatro diferentes actividades planeadas fueran realizadas utilizándose la aplicación Sketchfab (https://sketchfab.com/feed) para RA. Los temas contemplados con los contenidos en RA son dibujos de losas y vigas de hormigón reforzado, con enfoque en los detalles de dibujos para las barras de acero que son utilizadas para refuerzo del hormigón.

En las clases prácticas realizadas en el laboratorio de informática, se utilizaron los modelos tridimensionales disponibles en la plataforma Sketchfab web. En ese modelo había instrucciones adicionales para realizar las tareas solicitadas, por ejemplo, relacionar el refuerzo representado en el proyecto $2 \mathrm{D}$ con el correspondiente en el modelo 3D. Estas actividades se realizaron en el laboratorio accediéndose la plataforma Sketchfab. Las Figuras 2 y 3 presentan el modelo 3D para una viga. El modelo representado en la Figura 2 se accede a través del teléfono móvil. Por medio de la plataforma web se accede el modelo de la Figura 3.

Figura 2 - Modelo 3D en la aplicación Sketchfab para teléfono móvil

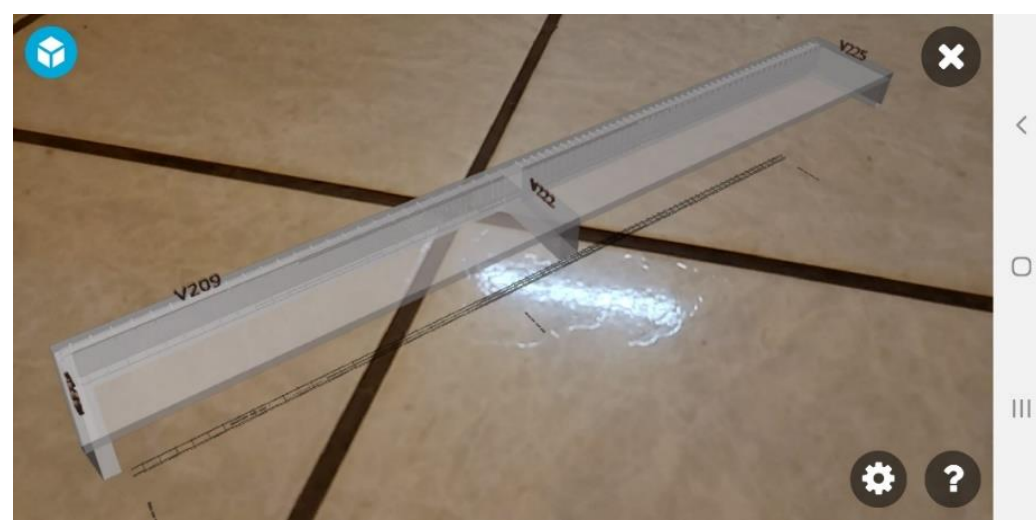

Fuente: Elaboración propia

Figura 3 - Modelo 3D en la plataforma web Sketchfab

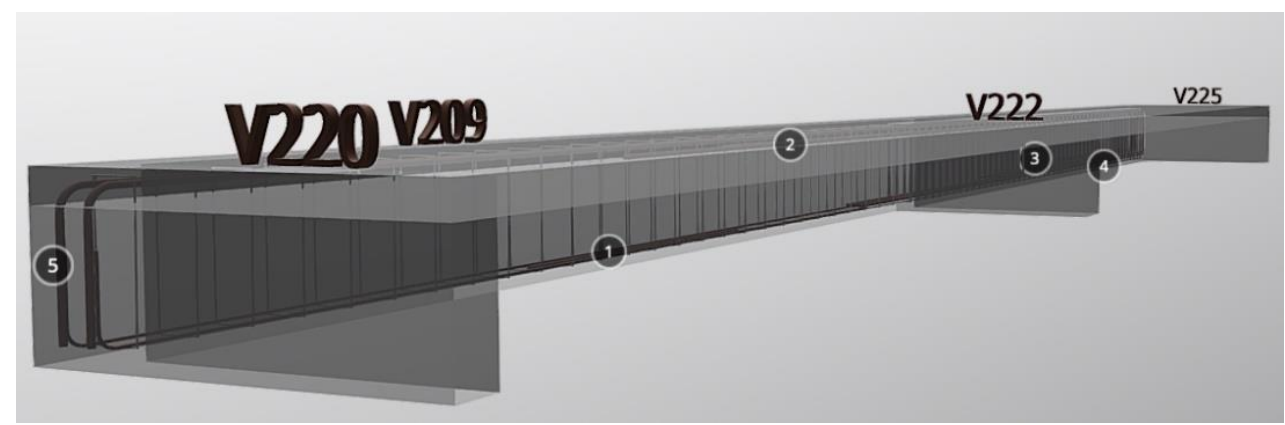

Fuente: Elaboración propia

Treinta y ocho alumnos respondieron a un cuestionario que mezcla tres preguntas para caracterización del alumno y veintitrés de escala Likert con 5 opciones. Así que el alumno debería elegir la opción 5 si estuviera totalmente de acuerdo con la afirmación y la 1 si estuviera totalmente en desacuerdo con la misma. 
Las veintitrés cuestiones de escala Likert fueron clasificadas de la siguiente manera: nivel de conocimiento del alumno acerca de los tópicos (2); experiencia del alumno con aplicaciones y recursos visuales para aprendizaje de ingeniería de estructuras (3); utilidad de los recursos y herramientas percibida por el alumno (7); facilidad de uso de los recursos y herramientas percibida por el alumno (6); y disfrute percibido e intención de utilizar nuevamente los recursos y herramientas (5).

\section{RESULTADOS}

De todos los treinta y ocho alumnos respondientes $24(63,2 \%)$ son de género masculino y $14(36,8 \%)$ de género femenino; 27 alumnos $(71,1 \%)$ tienen de 21 a 25 años y $11(28,9 \%)$ tienen más que 25 años. La mayoría $(60,5 \%)$ de los alumnos ya tuvieron contacto con actividades de dibujos de estructura por medio del trabajo de aprendiz o de técnico en edificaciones. La Tabla 1 muestra las ocurrencias de las 5 opciones de la escala Linkert para las afirmaciones sobre utilidad percibida (Q6 hasta Q11 y Q23) y disfrute percibido e intención de utilizarla (Q18 hasta Q22).

Tabla 1 - Resultado de las cuestiones de escala de Likert

\begin{tabular}{|c|l|c|c|c|c|c|c|}
\hline \multicolumn{2}{|c|}{ Afirmación } & \multicolumn{5}{c|}{ Escala Linkert } \\
\cline { 5 - 7 } \multicolumn{2}{|c|}{ (utilidad percibida) } & $\mathbf{5}$ & $\mathbf{4}$ & $\mathbf{3}$ & $\mathbf{2}$ & $\mathbf{1}$ \\
\hline Q6 & $\begin{array}{l}\text { Los modelos 3D ayudan en la visualización de los detalles de } \\
\text { las armaduras de las estructuras de hormigón reforzado. }\end{array}$ & 33 & 3 & 0 & 0 & 2 \\
\hline Q7 & $\begin{array}{l}\text { Creo que es muy relevante la utilización de recursos visuales } \\
\text { 3D para el aprendizaje de diseños de estructuras. }\end{array}$ & 34 & 1 & 2 & 0 & 1 \\
\hline Q8 & $\begin{array}{l}\text { La utilización de RA favorece la visualización de las } \\
\text { armaduras de los elementos estructurales de hormigón. }\end{array}$ & 31 & 4 & 2 & 0 & 1 \\
\hline Q9 & $\begin{array}{l}\text { Creo que es muy relevante la utilización de aplicaciones para } \\
\text { la enseñanza de diseños de estructuras de hormigón. }\end{array}$ & 32 & 3 & 2 & 0 & 1 \\
\hline Q10 & $\begin{array}{l}\text { La utilización de la herramienta RA influenció positivamente } \\
\text { en mi aprendizaje. }\end{array}$ & 28 & 4 & 5 & 0 & 1 \\
\hline Q11 & $\begin{array}{l}\text { Los modelos 3D son adecuados para la presentación del } \\
\text { contenido de la asignatura. }\end{array}$ & 31 & 6 & 0 & 0 & 1 \\
\hline Q23 & $\begin{array}{l}\text { Las herramientas RA podrían ser utilizadas en otras } \\
\text { asignaturas. }\end{array}$ & 30 & 7 & 0 & 0 & 1 \\
\hline \multicolumn{7}{|c|}{ (disfrute percibido e intención de utilizar los recursos) } \\
\hline Q18 & Es divertido utilizar la herramienta de RA. & 25 & 9 & 1 & 2 & 1 \\
\hline Q19 & Me sentí más motivado con las actividades RA. & 20 & 15 & 1 & 1 & 1 \\
\hline Q20 & $\begin{array}{l}\text { El uso de la herramienta RA hace que el aprendizaje sea más } \\
\text { interesante. }\end{array}$ & 28 & 7 & 1 & 1 & 1 \\
\hline Q21 & No me he aburrido utilizando la herramienta. & 23 & 9 & 4 & 0 & 2 \\
\hline Q22 & Me gustaría utilizar la herramienta en el futuro. & 29 & 6 & 2 & 0 & 1 \\
\hline
\end{tabular}

Fuente: Elaboración propia

El Gráfico 1 presenta los resultados, en porcentajes, para las cuestiones sobre la utilidad de los recursos y herramientas percibida por el alumno. En el Gráfico 2 se tienen los porcentajes de los resultados de las cuestiones sobre el disfrute percibido y la intención de utilizar los recursos y herramientas nuevamente. 
Gráfico 1 - Resultado de las cuestiones de escala de Likert

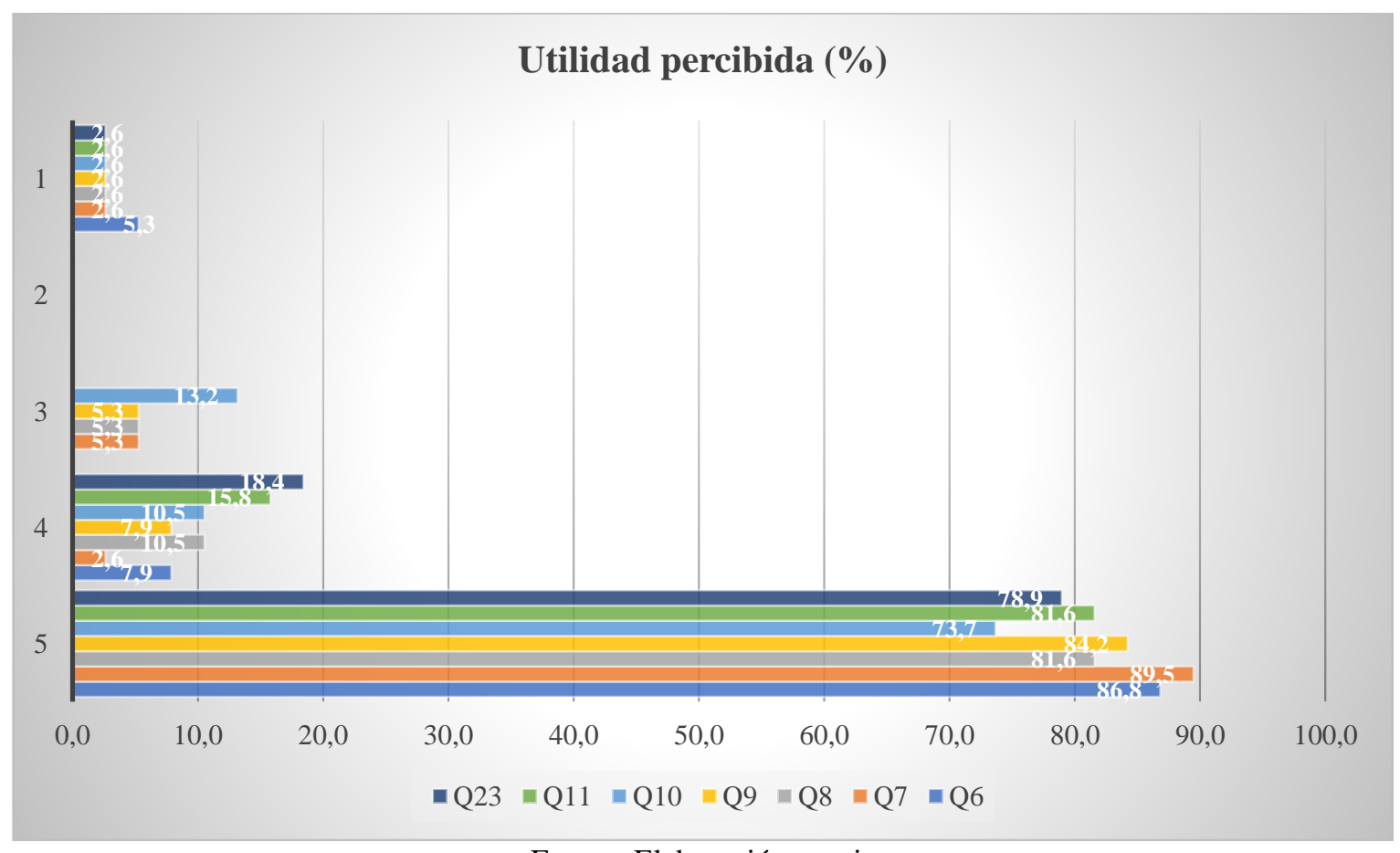

Fuente: Elaboración propia

Gráfico 2 - Resultado de las cuestiones de escala de Likert

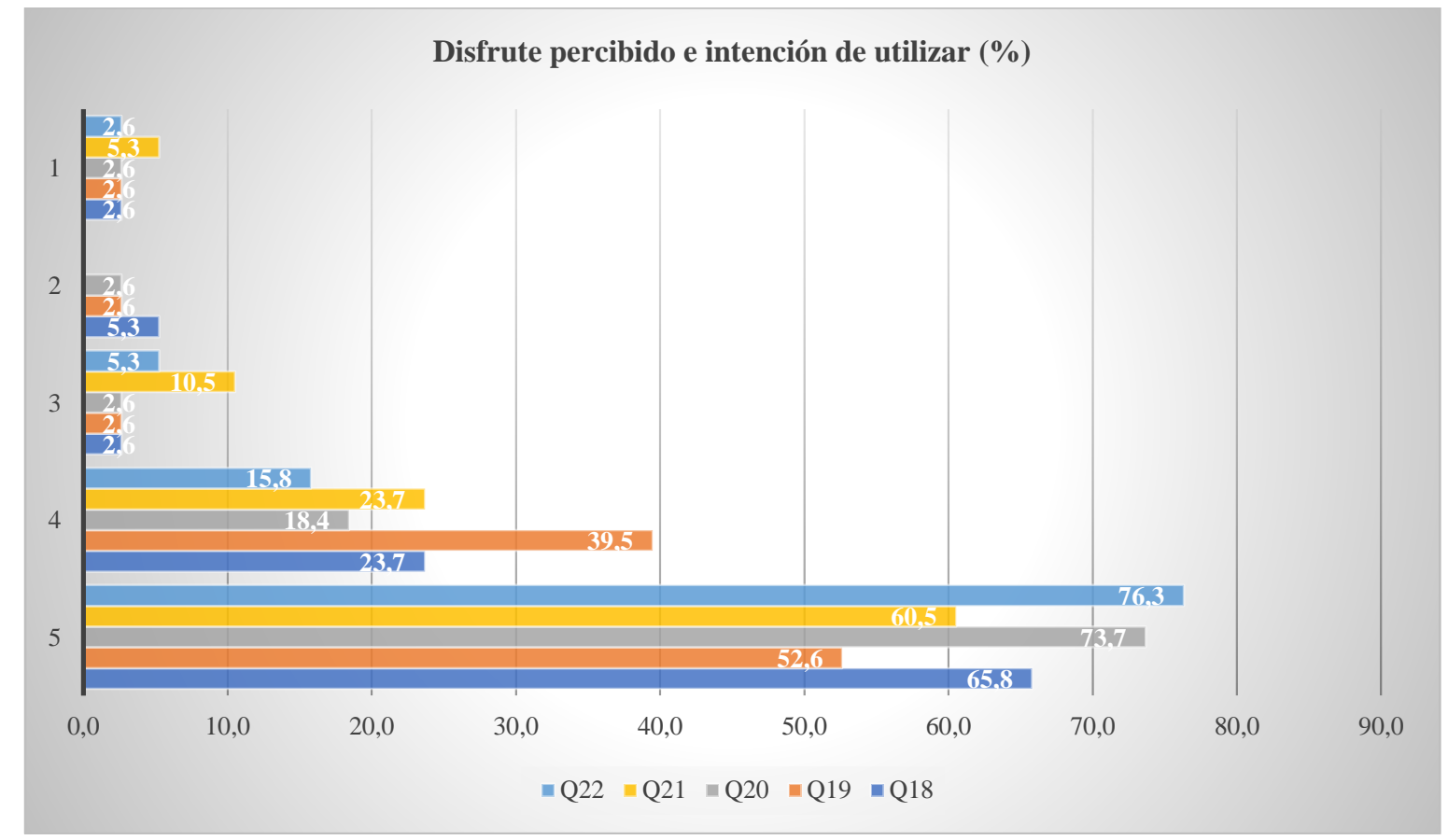

Fuente: Elaboración propia 
Para las afirmaciones del grupo de utilidad percibida (Gráfico 1), más de noventa por ciento de los alumnos eligieron las opciones 4 o 5, con excepción de la Q10 (84,2\%), lo que indica una gran utilidad de los recursos percibida por ellos. Lo mismo ocurrió con las respuestas a las cuestiones del grupo de disfrute percibido e intención de utilizar los recursos donde más de $80 \%$ de los alumnos concordaron con las afirmaciones.

A los alumnos, no solo les gustó las actividades, sino que también se sintieron motivados con la utilización de RA para el aprendizaje de los contenidos de esa asignatura. Eso se ve en las respuestas de los dos grupos de afirmaciones. Además, a los 92,1\% de los alumnos les gustaría utilizar la herramienta nuevamente (Q22). También casi todos ellos $(97,4 \%)$ recomendaron el empleo de RA en otras asignaturas del curso (Q23), lo que confirma la relevancia de los modelos 3D para motivar el aprendizaje de ingeniería civil, y de la tecnología aquí empelada.

\section{CONSIDERACIONES FINALES}

Basándose en las respuestas de los alumnos, se cree que es positiva y relevante la utilización de recursos 3D por medio de RA, para mejorar la habilidad de visualización espacial (HVE) y por consecuencia el desempeño del alumno. Pero eso fue un estudio preliminar para probar el uso de RA en las asignaturas de estructuras de hormigón. La propuesta fue planear cuatro actividades con los recursos de RA y evaluar la percepción del alumno sobre la utilidad de los recursos y herramientas, la facilidad de uso de ellos y el disfrute e intensión de utilizar los recursos nuevamente. Los resultados permitirán las adaptaciones necesarias para el empleo del recurso en otras clases y asignaturas. El estudio puso de manifiesto que, con la plataforma utilizada, Sketchfab, se lleva mucho tiempo para procesar modelos con gran cantidad de objetos cuando se usa el teléfono móvil. Aunque se haya confirmado la declaración de Meža, Turk y Dolenc (2015) sobre una de las principales barreras para el uso de RA con fines educativos: el tamaño de los modelos 3D, la aplicación elegida se presenta adecuada para los modelos más simples como los que fue presentados a los alumnos y que son suficientes para comprensión del contenido elegido.

También se obtuvo la necesidad de contar con la disponibilidad de los dispositivos adecuados para poder llevar a cabo la experiencia. Mismo con la limitación de disponibilidad de dispositivos adecuados, ni todos los alumnos tenían el móvil con la configuración necesaria, se puede ver en el análisis de los resultados del cuestionario, que el uso de RA para la visualización tridimensional es motivador para los estudiantes, ya que facilitan la comprensión de la distribución del refuerzo dentro de los elementos estructurales, permitiendo la realización de las actividades de una manera más divertida.

Esta investigación pone de manifiesto, y coincide con los resultados obtenidos por otros autores (BARROSO, CABERO y MORENO, 2016; MARÍN, 2017; BARROSO, CABERO y GUTIÉRREZ, 2018; MARTÍNEZ y FERNÁNDEZ, 2018; CABERO y ROIG, 2019), donde fue utilizada con estudiantes universitarios de diferentes disciplinas, desde Medicina a Bellas Artes y Ciencias de la Educación. Por tanto, podemos señalar que el grado de aceptación de esta tecnología por los estudiantes, es bastante significativo, lo que nos lleva a su recomendación para la formación.

\section{Agradecimientos}

Se agradece especialmente a la directoria y demás miembros del Secretariado de Recursos Audiovisuales y Nuevas Tecnologías de la Universidad de Sevilla (España) quienes han compartido sus experiencias y a la PUC Minas por el apoyo para estadía de la profesora Gláucia en Sevilla (2018-2019). 


\section{REFERENCIAS}

ABDULLAH, F.; WARD, R. Developing a General Extended Technology Acceptance Model for E-Learning (GETAMEL) by analysing commonly used external factors. Computers in Human Behavior, v.56, p. 238-256, 2016. https://doi.org/10.1016/j.chb.2015.11.036

AL-EMRAN, M.; MEZHUYEV, V.; KAMALUDIN, A. Technology Acceptance Model in M-learning context: A systematic review. Computers \& Education, v.125, p. 389-412, 2018. https://doi.org/10.1016/j.compedu.2018.06.008

AZUMA, R.T. A survey of augmented reality. Presence-Teleoperators and Virtual Environments, v.6, n.4, p. 355-385, 1997.

BARROSO, J.; CABERO, J.; MORENO, A.M. La utilización de objetos de aprendizaje en realidad aumentada en la enseñanza de la Medicina. Innoeduca International Journal of Technology and Educational Innovation, v.2, n.2, p. 77-83, 2016.

BARROSO, J.; CABERO, J.; GUTIÉRREZ, J.J. La producción de objetos de aprendizaje en realidad aumentada por estudiantes universitarios grado de aceptación de esta tecnología y motivación para su uso. Revista Mexicana de Investigación Educativa, v.23, n.79, p.12611283, 2018.

CABERO, J.; BARROSO, J. The educational possibilities of Augmented Reality. New Approaches in Educational Research, v.5, n.1, p. 44-50, 2016. https://doi.org/10.7821/naer.2016.1.140

CABERO, J.; PÉREZ, J. L. Validación del modelo TAM de adopción de la Realidad Aumentada mediante ecuaciones estructurales. Estudios sobre Educación, v.34, p. 129-153, 2018. https://doi.org/10.15581/004.34.129-153

CABERO, J.; ROIG, R. The Motivation of Technological Scenarios in Augmented Reality (AR): Results of Dierent Experiments. Applied Sciences, v.9, 2019. https://doi.org/10.3390/app9142907

DAVIS, F. D. Perceived usefulness, perceived ease of use, and user acceptance of information technology. MIS Quarterly, v.13, p. 319-340, 1989.

FOGARTY, J.; MCCORMICK, J.; EL-TAWIL S. Improving Student Understanding of Complex Spatial Arrangements with Virtual Reality. Journal of Professional Issues in $\begin{array}{lllllll}\text { Engineering Education and Practice, v.144, n.2, p. } 1-10, & 2018 .\end{array}$ https://doi.org/10.1061/(ASCE)EI.1943-5541.0000349

LIN, H. Influence of design training and spatial solution strategies on spatial ability performance. International Journal of Technology \& Design Education, v.26, p. 123-131, 2016. https://doi.org/10.1007/s10798-015-9302-7 
MARÍN, V. The augmented reality in the educational sphere of student of degree in chilhood education. Case Study. Pixel-Bit. Revista de Medios y Educación, v.51, p. 7-19, 2017. http://dx.doi.org/10.12795/pixelbit.2017.i51.01

MARTÍNEZ, S.; FERNÁNDEZ, B. Objetos de Realidad Aumentada: percepciones del alumnado de Pedagogía. Pixel-Bit. Revista de Medios y Educación, v.53, p. 207-220, 2018. http://dx.doi.org/10.12795/pixelbit.2018.i53.14

MELLO, G. N. A. Teachers' perspective about factors that prevent success in teaching and learning process in higher education of engineering in Brazil. Problems of Education in the 21st Century, v.74, p. 61-70, 2016. Disponible en: http://bit.ly/350Os5X. Acceso en: 25 jul. 2020.

MELLO, G. N. A.; MAIA, E.V.; CALIXTO, J. M. F. CONCWEB: hybrid learning tool for reinforced concrete design. ETD-Educação Temática Digital, v.18, n.1, p. 156-177, 2016. https://doi.org/10.20396/etd.v18i1.8638248

MEŽA, S.; TURK, Ž.; DOLENC, M. Measuring potential of augmented reality in civil engineering. Advances in Engineering Software, v.90, p. 1-10, 2015. https://doi.org/10.1016/j.advengsoft.2015.06.005

MOLYNEAUX, T. et al. An evaluation of the learning of structural engineering concepts during the first two years of a project-based engineering degree. European Journal of Engineering Education, v.32, n.1, p. 01-08, 2007. Disponible en: http://bit.ly/2YH0krD. Acceso en: 25 jul. 2020.

SEGIL, J. L. et al. Investigation of spatial visualization skills across world regions. Frontiers in Education Conference, p. 18-21, 2017. Disponible en: http://bit.ly/2P5ORP9. Acceso en: 25 jul. 2020.

SORBY, S. A. A course in spatial visualization and its impact on the retention of female engineering students. Journal of Women and Minorities in Science and Engineering, v.7, p. 153-172, 2001.

SORBY, S. A.; VEURINK, N. Spatial skills among minority and international engineering students. American Society for Engineering Education, v.25, 2012. Disponible en: http://bit.ly/2PwLMqf. Acceso en: 25 jul. 2020.

\section{STUDENTS' PERCEPTION ABOUT THE USE OF AUGMENTED REALITY IN REINFORCED CONCRETE DESIGN COURSE}

Abstract: The design is one of the main activities of Civil Engineer, so his involvement with drawings and representation of structures is intense. In this way, the spatial visualization competence and mental rotation is especially important for that professional. The motivation of this research was to plan and develop activities using a mobile application with augmented reality resources, for the disciplines of reinforced concrete design in the Civil Engineering course. Four activities were developed using the augmented reality resources with the 
Sketchfab platform. Thirty-eight students did the activities and answered a questionnaire that was used as an instrument to assess the students' perception of the usefulness, ease of use, motivation, and intention to use the resources for learning the content. Almost all students believe that the resources used are truly relevant to learning, make learning more fun and facilitate the visualization of the details of the structures. In this context, it was concluded that, while there is still a lot of research to be done, augmented reality is an important resource to improve the spatial visualization skill of engineering students and consequently the performance of structures.

Keywords: Structural design. Reinforced concrete. Spatial visualization. Augmented reality. 